\title{
Erratum to: Comparative evaluation of the diagnosis, reporting and investigation of malaria cases in China, 2005-2014: transition from control to elimination for the national malaria programme
}

\author{
Jun-Ling Sun ${ }^{1 \dagger}$, Sheng Zhou ${ }^{1 \dagger}$, Qi-Bin Geng ${ }^{1,2+}$, Qian Zhang ${ }^{1}$, Zi-Ke Zhang ${ }^{1,3}$, Can-Jun Zheng ${ }^{1}$, Wen-Biao Hu ${ }^{4}$,
} Archie C.A. Clements ${ }^{5}$, Sheng-Jie Lai ${ }^{1,6}$ and Zhong-Jie Li ${ }^{1 *}$

\section{Erratum}

After publication of this article [1] it was noticed that the wrong figure was used for Fig. 3. Please see the correct Fig. 3 below.

\section{Author details \\ 'Division of Infectious Diseases, Key Laboratory of Surveillance and Early-warning on Infectious Disease, Chinese Center for Disease Control and Prevention, 155 Changbai Road, Changping District, Beijing 102206, China. ${ }^{2}$ State Key Laboratory of Virology and College of Life Sciences, Wuhan University, Wuhan 430072, China. ${ }^{3}$ Center of Clinical Laboratory, First Affiliated Hospital, College of Medicine, Zhejiang University, Hangzhou, China. ${ }^{4}$ School of Public Health and Social Work, Queensland University of Technology, Brisbane, Australia. ${ }^{5}$ Research School of Population Health, College of Medicine, Biology and Environment, The Australian National University, Canberra, Australia. ${ }^{6}$ Department of Geography and Environment, University of Southampton, Southampton SO17 1BJ, UK.}

Received: 22 May 2017 Accepted: 22 May 2017

Published online: 19 June 2017

\section{Reference}

1. Sun J-L, Zhou S, Geng Q-B, Zhang Q, Zhang Z-K, Zheng C-J, et al.

Comparative evaluation of the diagnosis, reporting and investigation of malaria cases in China, 2005-2014: transition from control to elimination for the national malaria programme. Infect Dis Poverty. 2016;5:65.

\footnotetext{
*Correspondence: lizhongjiecdc@163.com

${ }^{\dagger}$ Equal contributors

'Division of Infectious Diseases, Key Laboratory of Surveillance and Early-warning on Infectious Disease, Chinese Center for Disease Control and Prevention, 155 Changbai Road, Changping District, Beijing 102206, China Full list of author information is available at the end of the article
} 

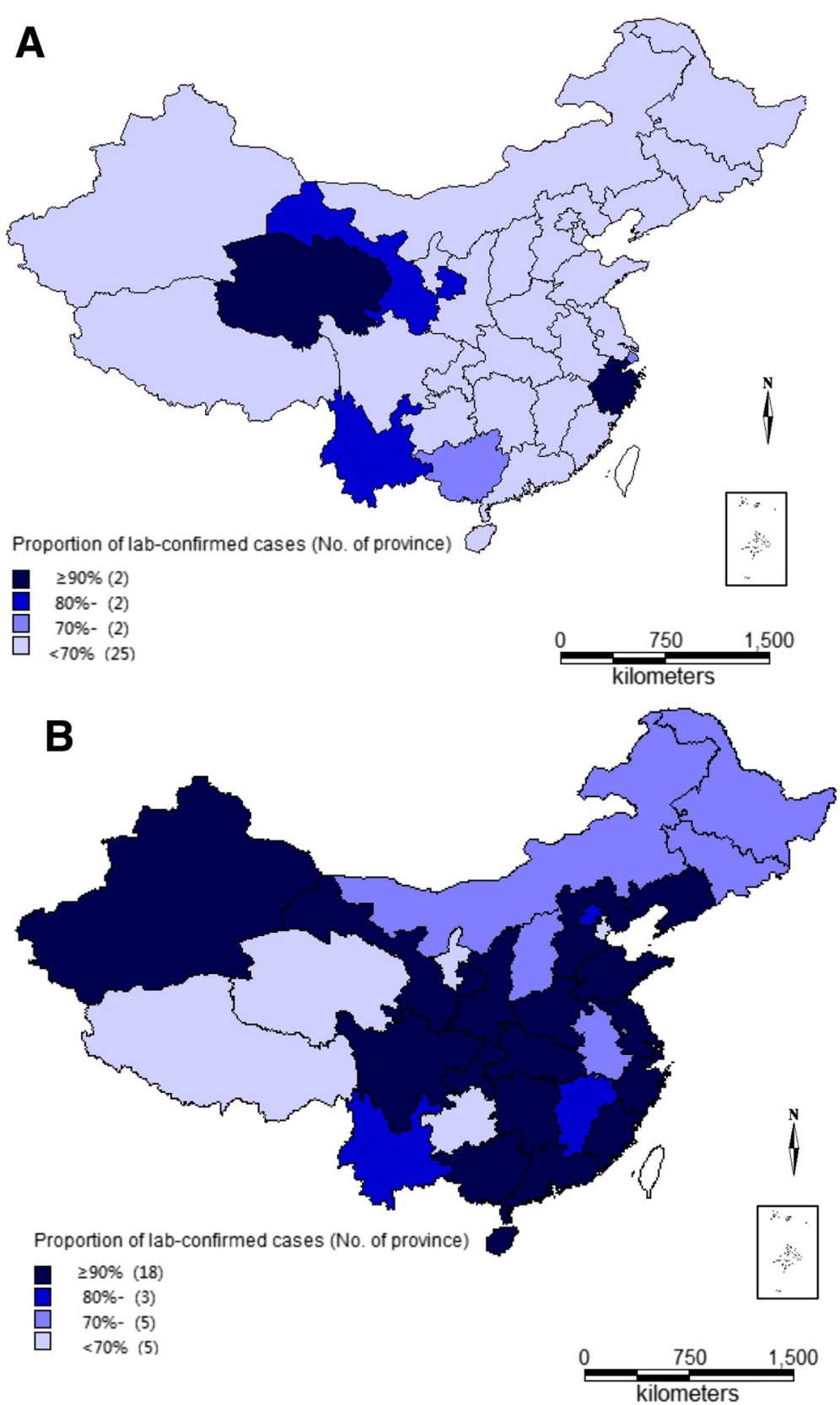

Fig. 3 Proportion of lab-confirmed malaria by province during 2005-2014 in China (a control stage [2005-2010]; b elimination stage [2011-2014])] 